\title{
A Small Ku-Band Polarization Tracking Active Phased Array for Mobile Satellite Communications
}

\author{
Wei Shi, ${ }^{1,2}$ Zuping Qian, ${ }^{1}$ Jun Zhou, ${ }^{3,4}$ Xinbo Qu, ${ }^{2}$ Yang Xiang, ${ }^{2}$ and Liu Hong ${ }^{2}$ \\ ${ }^{1}$ Institute of Communication Engineering, PLA University of Science and Technology, Nanjing 210016, China \\ ${ }^{2}$ Antenna Research Laboratory, Nanjing Telecommunication Technology Institute, Nanjing 210007, China \\ ${ }^{3}$ State Key Laboratory of Millimeter Waves, Southeast University, Nanjing 210096, China \\ ${ }^{4}$ Department of Micro-systems, Nanjing Electronic Device Institute, Nanjing 210096, China \\ Correspondence should be addressed to Wei Shi; sw_ant@sina.com
}

Received 19 April 2013; Revised 21 September 2013; Accepted 22 September 2013

Academic Editor: Z. N. Chen

Copyright (c) 2013 Wei Shi et al. This is an open access article distributed under the Creative Commons Attribution License, which permits unrestricted use, distribution, and reproduction in any medium, provided the original work is properly cited.

\begin{abstract}
A compact polarization tracking active phased array for Ku-band mobile satellite signal reception is presented. In contrast with conventional mechanically tracking antennas, the approach presented here meets the requirements of beam tracking and polarization tracking simultaneously without any servo components. The two-layer stacked square patch fed by two probes is used as antenna element. The impedance bandwidth of $16 \%$ for the element covers the operating frequency range from $12.25 \mathrm{GHz}$ to 12.75 GHz. In the presence of mutual coupling, the dimensional parameters for each element of the small $7 \times 7$ array are optimized during beam scanning and polarization tracking. The compact polarization tracking modules based on the low-temperature cofired ceramic (LTCC) system-in-package ( $\mathrm{SiP}$ ) technology are proposed. A small active phased array prototype with the size of $120 \mathrm{~mm}$ (length) $\times 120 \mathrm{~mm}$ (width) $\times 55 \mathrm{~mm}$ (height) is developed. The measured polarization tracking patterns of the prototype are given. The polarization tracking beam can be steered in the elevation up to $50^{\circ}$. The gain of no less than $16.0 \mathrm{dBi}$ and the aperture efficiency of more than $50 \%$ are obtained. The measured and simulated polarization tracking patterns agreed well.
\end{abstract}

\section{Introduction}

Geostationary satellite communication is widely used in military and civilian fields for wide area coverage. In order to improve communication capacity, Ku-band satellite communication system utilizes orthogonal linear polarization transmission in both uplink $(14.0 \mathrm{GHz} 14.5 \mathrm{GHz})$ and downlink $(12.25 \mathrm{GHz} \sim 12.75 \mathrm{GHz})$ for frequency reuse. The mobile antenna terminals may not point to the satellite when there is the rapid change in azimuth, pitch, and roll of vehicles, airplanes, and ships. One challenge for mobile antenna terminals in $\mathrm{Ku}$-band is to avoid polarization mismatch. Therefore, the mobile antenna terminals in Ku-band should meet the requirements of beam tracking and polarization tracking simultaneously.

Reflector or lens antenna is used most widely for its high efficiency and low cost [1-3]. The beam pointing and polarization are steered mechanically. But the requirement for low profile and high tracking speed is not easily met.
The mechanically tracking array antenna of stair structure has lower profile $[4,5]$, but the low radiating efficiency and relatively higher sidelobe limit its use in some applications. The hybrid tracking planar antenna combines mechanical tracking in azimuth and electronic tracking in elevation $[6,7]$. However, it is difficult to integrate the mechanical servo components with the top surface of the vehicles, airplanes, and ships.

This paper presents a new polarization tracking active phased array based on LTCC-SiP polarization tracking module. The beam and the orientation of the linear polarization can be electronically steered simultaneously [8]. The radiating element is based on a stacked square microstrip patch orthogonally fed by two probes. Each probe excites horizontally or vertically polarized wave. Therefore, the arbitrary linear polarization can be obtained through the combination of two orthogonal polarizations. We have designed a new compact polarization tracking module with the LTCC-SiP technology, which can be used to reduce the height of 


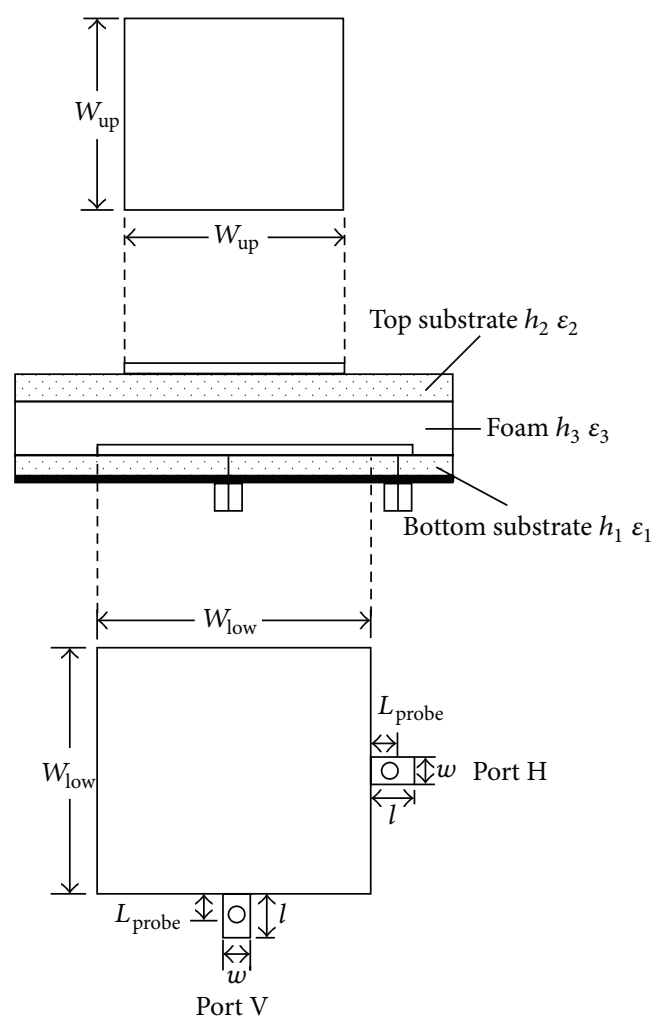

FIgURE 1: Configuration of the antenna element fed by two orthogonal probes.

the array. The phase and polarization of the radiating element can be electronically controlled by the phase settings of the module.

A model for the small $7 \times 7$ array was established using CST Microwave Studio. We analyzed the impedance variation of central element during beam scanning and polarization tracking in the presence of mutual coupling. Based on the simulated results, a small polarization tracking active phased array prototype for reception in the frequency range from $12.25 \mathrm{GHz}$ to $12.75 \mathrm{GHz}$ was developed. The height of the array is only $55 \mathrm{~mm}$. In the microwave chamber, the measured radiation patterns for vertical, horizontal, and diagonal polarization were obtained via phase settings of the proposed polarization tracking modules. The beam can be electronically steered up to $50^{\circ}$ in the elevation. The developed prototype has the minimum gain of $16.0 \mathrm{dBi}$ and the aperture efficiency of $50 \%$. The measured polarization tracking patterns and the simulated ones agree well, which demonstrates the usefulness of the design method based on mutual coupling.

\section{Array Design Based on Mutual Coupling}

The arbitrary linearly polarized wave can be decomposed into vertically and horizontally polarized component with the same phase and different amplitude. Thus, the radiating element with orthogonal dual ports radiates vertically and horizontally polarized wave simultaneously. The weighted coefficients for the dual ports of the radiating element can be controlled via the phase settings of polarization tracking module, and the linear polarization of arbitrary orientation can be obtained. Thus, the antenna array has the capability of polarization tracking without mechanical rotation of the whole array.

The cross-dipoles can be used as the radiating element for polarization tracking phased array [9], but the height of the element is $0.48 \lambda_{0}$. The dual aperture coupled microstrip antenna can also be used to radiate orthogonally polarized waves $[3,7]$. However, the coaxial connecters of the microwave modules result in the difficulty of connections between the aperture-coupled antenna elements and the modules. For low profile, easy fabrication, and low cost, twolayer stacked square microstrip antenna with dual probes is used as radiating element, as shown in Figure 1. Broad bandwidth can be obtained with electromagnetic coupling mechanism between top and bottom patches. Arlon Diclad 880 with $\varepsilon_{r}=2.2$ and $h=0.508 \mathrm{~mm}$ is used for top and bottom substrates. Rohacell HF51 with $\varepsilon_{r}=1.05$ and $h=$ $1.5 \mathrm{~mm}$ is used for the foam. With the symmetrical structure of the square patch, port $\mathrm{H}$ and port $\mathrm{V}$ approximately have the same radiation pattern and resonance frequency. Therefore, the little effect of the patterns on the polarization combination can be neglected, and the amplitude and phase weighted coefficients of dual ports determine the final polarization of the radiating element.

The square grid arrangement for the radiating elements is used for the simplicity of the feed network. We designed a small $7 \times 7$ array shown in Figure 2, which may be equivalent 


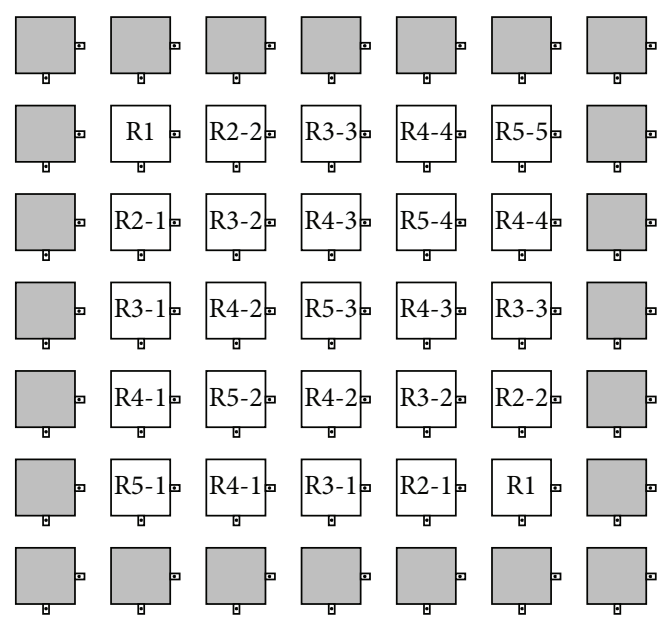

(a) Layout

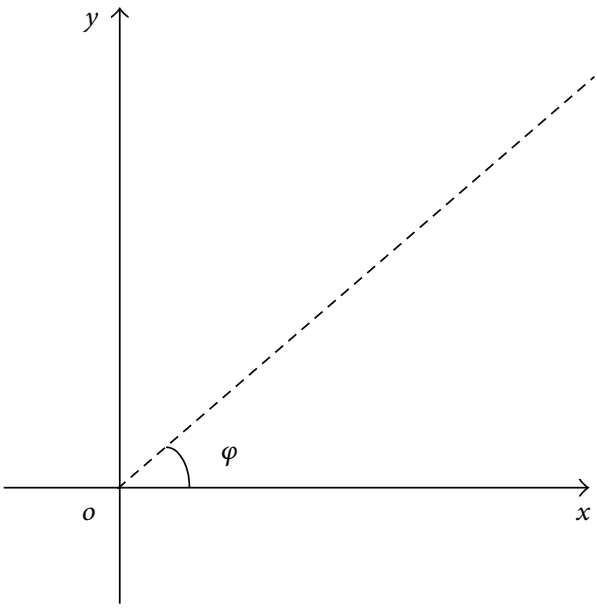

(b) Coordinate system

FIGURE 2: Topology of the small array (the outermost elements with gray color indicate passive elements terminated with $50 \Omega$ matched loads).

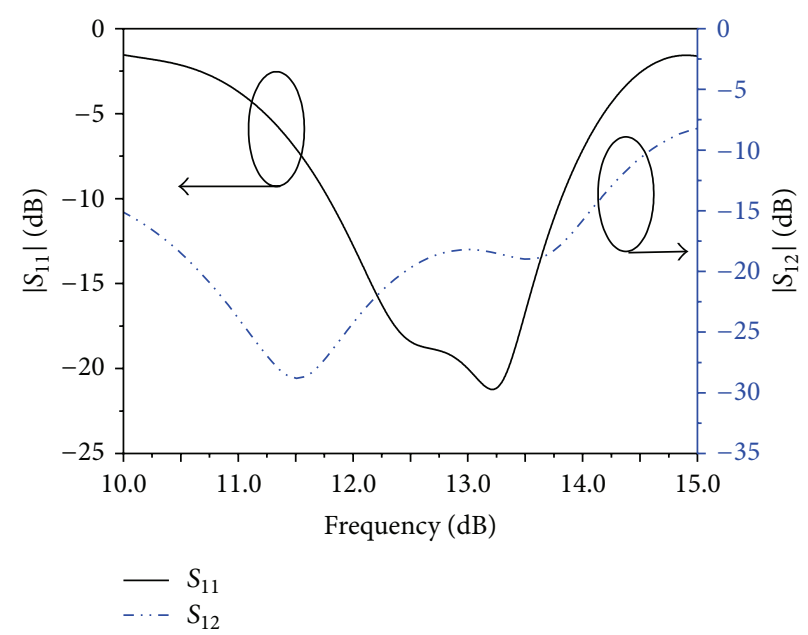

FIGURE 3: The simulated $S_{11}$ and $S_{12}$ of isolated antenna element.

to a large array for the center element R5-3. Thus, the effect of mutual coupling on the impedance of radiating element, as well as active element pattern, can be analyzed. The radiating elements are spaced a distance of $0.5 \lambda_{0}$, wherein $\lambda_{0}$ is the wavelength in free space at $12.5 \mathrm{GHz}$. The outermost elements with gray color are terminated with loads of $50 \Omega$ to form passive elements, which are used to solve the problem of deterioration of edge element pattern of the inner $5 \times 5$ active array.

We used software CST Microwave Studio to design the isolated radiating element. Array A in Table 1 was formed by 7 rows of 7 elements designed without considering mutual coupling. The structural symmetry of isolated element contributes to the same simulated reflection coefficients of dual ports shown in Figure 3. The simulated $10 \mathrm{~dB}$ impedance bandwidth $\left(\left|S_{11}\right|<-10 \mathrm{~dB}\right)$ was found to be $16 \%(1.8 \mathrm{GHz}$ 13.8 GHz), which is sufficient for Ku-band satellite receiver system. Furthermore, the coupling coefficient between port
TABLE 1: The structural parameters for the radiating element (unit: $\mathrm{mm})$.

\begin{tabular}{lccccc}
\hline Element parameters & $W_{\text {up }}$ & $W_{\text {low }}$ & $w$ & $l$ & $L_{\text {probe }}$ \\
\hline Array A (initial design ) & 7.5 & 7.8 & 1.4 & 1.05 & 0.4 \\
Array B (final design ) & 6.6 & 7.9 & 0.85 & 1.05 & 0.4 \\
\hline
\end{tabular}

$\mathrm{H}$ and port $\mathrm{V}$ is below $-18 \mathrm{~dB}$. However, the difficult challenge with the polarization tracking phased array is the mutual coupling, which will result in the variation of the port impedance of each element during polarization tracking and beam scanning. Thus, the design parameters in Table 1 for the radiating element in array A may be not optimum due to the presence of mutual coupling thereby degrading the array performance.

A small array model was established in CST Microwave Studio for mutual coupling analysis. If the amplitude 


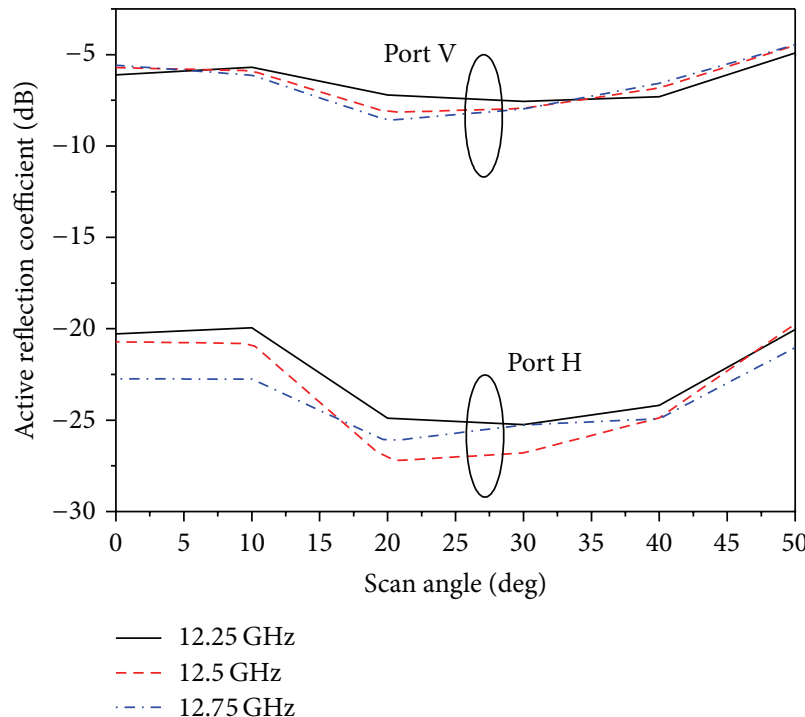

(a) Array A

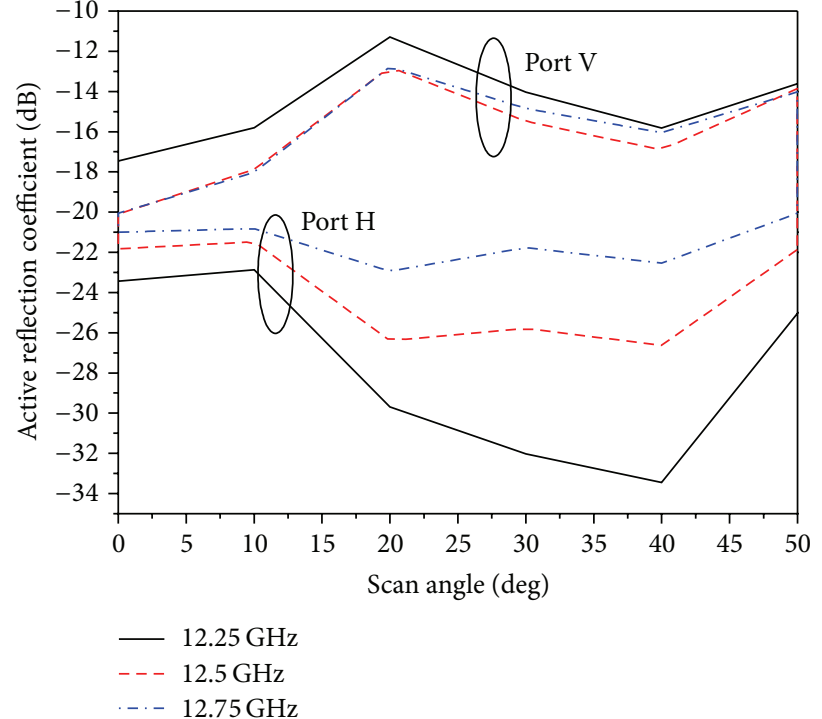

(b) Array B

FIGURE 4: The active reflection coefficient of central element R5-3 (azimuth angle $\varphi=0^{\circ}$, vertical polarization).

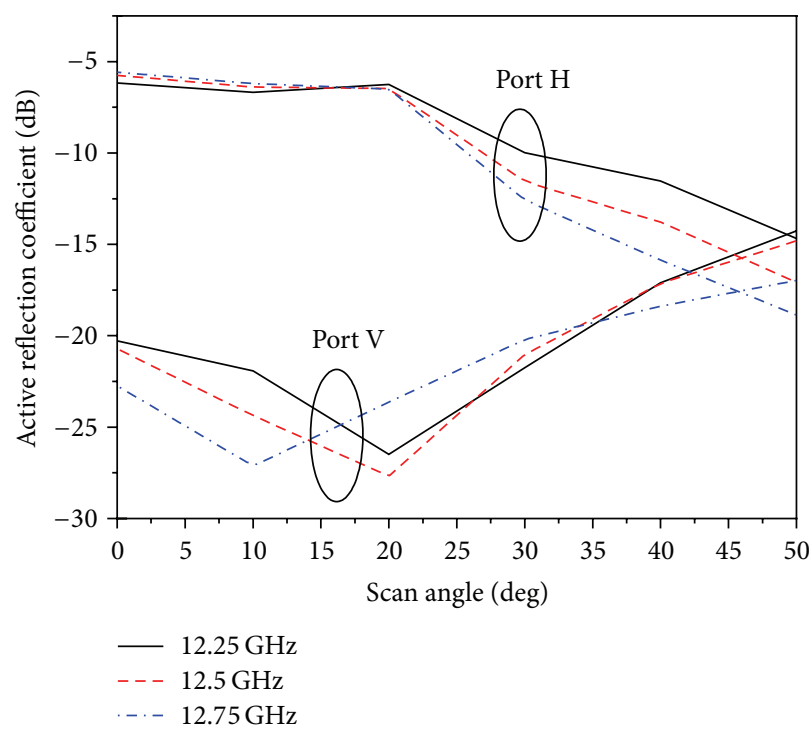

(a) Array A

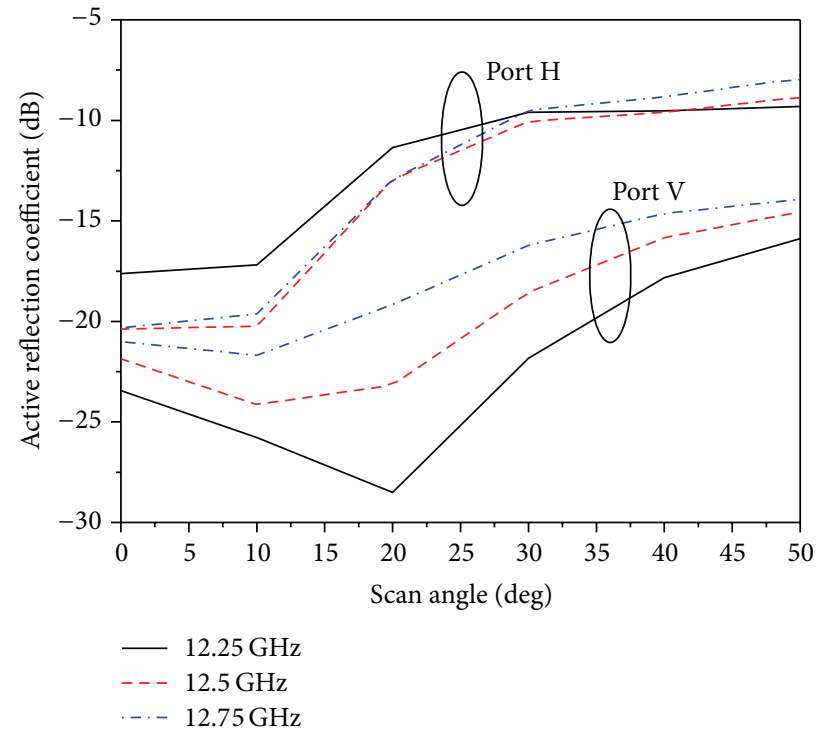

(b) Array B

FIGURE 5: The active reflection coefficient of central element R5-3 (azimuth angle $\varphi=0^{\circ}$, horizontal polarization).

weighted coefficients for the orthogonal ports (port $\mathrm{H}$ and port $\mathrm{V})$ are $(1,0),(0,1)$, and $(1,1)$, respectively, the small array will radiate vertically, horizontally, and diagonally polarized wave. In each polarization, the beam can be steered from $0^{\circ}$ to $50^{\circ}$ in elevation with phase variation between antenna elements. The key dimensional parameters $W_{\text {up }}, W_{\text {low }}$, and $w$ can be adjusted via simulation to mitigate the mutual coupling effect on the variation of port impedance during polarization tracking and beam scanning. The new parameters of radiating elements proposed for array $\mathrm{B}$ are given in Table 1 . The variations of active reflection coefficient of central element R5-3 in both array A and array $\mathrm{B}$ are given in Figures 4, 5, and 6. Compared with array A, the active reflection coefficient of central element R5-3 in array $B$ has been reduced generally when the polarization tracking beam is steered in the $x z$-plane $\left(\varphi=0^{\circ}\right)$ within the scan range of $\pm 25^{\circ}$. As the scan angle increases, the performance of active reflection coefficient of array $\mathrm{B}$ degrades gradually, which is obvious especially for the diagonal polarization. When the beam is steered to $50^{\circ}$ in elevation, the active reflection coefficient of about $-7 \mathrm{~dB}$ in the frequency range can be achieved for port $\mathrm{H}$. The gain versus scan angle of both array 


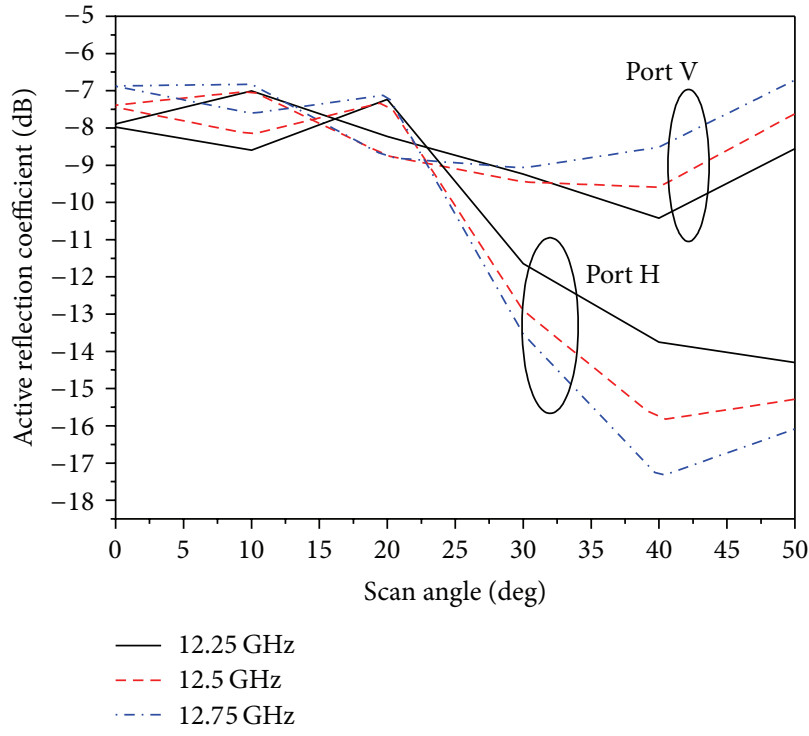

(a) Array A

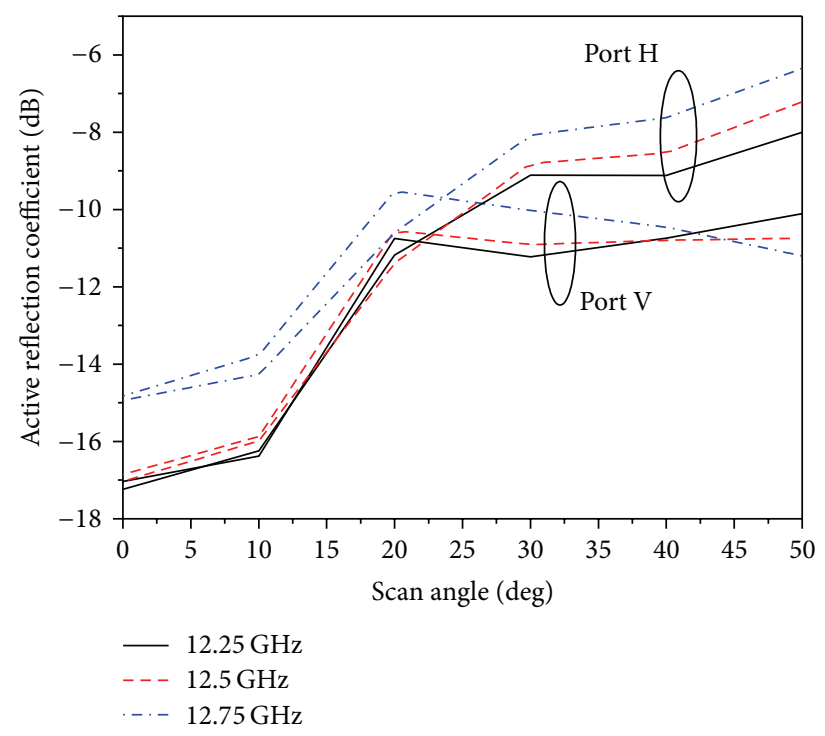

(b) Array B

FIGURE 6: The active reflection coefficient of central element R5-3 (azimuth angle $\varphi=0^{\circ}$, diagonal polarization).

A and array B was computed for comparison. The simulated results for three typical polarizations are shown in Figure 7. The gain improvement for array $\mathrm{B}$ can be observed.

As shown in Figure 8, a small $7 \times 7$ element array was fabricated based on the dimensional parameters of array $\mathrm{B}$. The active element patterns of central element R5-3 were measured when all of the other ports except for port $\mathrm{V}$ were terminated with passive loads of $50 \Omega$. With reference to Figure 2, the $x z$-plane $\left(\varphi=0^{\circ}\right)$ is the H-plane for port V, and the $y z$-plane $\left(\varphi=90^{\circ}\right)$ is the E-plane. The active element patterns of central element R5-3 in H- and E-planes are given in Figure 9. In the area of the main lobe, the agreement between the simulated and measured results can be observed. Both the simulated and measured results indicate the half-power beamwidth of approximately $120^{\circ}$ and $112^{\circ}$ in the H-plane and the E-plane, respectively. No blind spot is observed. Due to the approximate symmetry in structure, the active element pattern of port $\mathrm{H}$ has the similarity with that of port $\mathrm{V}$.

\section{Polarization Tracking Module}

The polarization tracking modules are key components for the polarization tracking active phased array. The dual input ports shown in Figure 10(b) are connected to the orthogonal dual ports of antenna element. The dual inputs from port $\mathrm{V}$ and port $\mathrm{H}$ can be combined into single output in phase via differential phase setting $\Delta \varphi$. Thus, the polarization mismatch with incoming wave from satellite can be avoided. Figure 10(a) shows the coordinate system based on the unit vectors of orthogonal polarizations, wherein $\vec{v}$ and $\vec{h}$ denote the vertical and horizontal polarizations, respectively. The orientation of polarization for the incoming wave is denoted by $\vec{p}$, and the included angle formed by $\vec{p}$ and $\vec{v}$ is denoted by $\vec{\alpha}$. If we neglect the effects of radiation patterns of dual ports, the inputs of the Lange coupler are obtained as follows.

Port (3):

$$
u_{3}=a \sqrt{G_{\mathrm{amp}}} \cos \alpha
$$

Port (4):

$$
u_{4}=a \sqrt{G_{\mathrm{amp}}} \sin \alpha
$$

wherein $G_{\mathrm{amp}}$ is the gain of the LNA. Assuming that the two channels are consistent, the signal at the output of the receiver module can be yielded as

$$
\begin{aligned}
u_{c}= & \frac{a}{2 \sqrt{L_{T}}} \sqrt{G_{\mathrm{amp}}} \exp \left[j\left(180^{\circ}-\alpha+\varphi_{0}\right)\right] \\
& +\frac{a}{2 \sqrt{L_{T}}} \sqrt{G_{\mathrm{amp}}} \exp \left[j\left(90^{\circ}+\varphi_{0}+\Delta \varphi+\alpha\right)\right] .
\end{aligned}
$$

Herein, $L_{T}$ is the average insertion loss in each channel. If the phase factor in (2) satisfies the condition as follows,

$$
\Delta \varphi=90^{\circ}-2 \alpha
$$

the combined signal has no polarization mismatch loss. In this case, the maximum combined signal is given as

$$
u_{c}=\frac{a}{\sqrt{L_{T}}} \sqrt{G_{\mathrm{amp}}} \exp \left[j\left(180^{\circ}-\alpha+\varphi_{0}\right)\right] .
$$

Based on LTCC technology, a vertical transition from coplanar waveguide to stripline is presented to reduce the size of the module [10]. Figure 11 shows the vertical transition configuration and fabricated prototype. The simulated and 


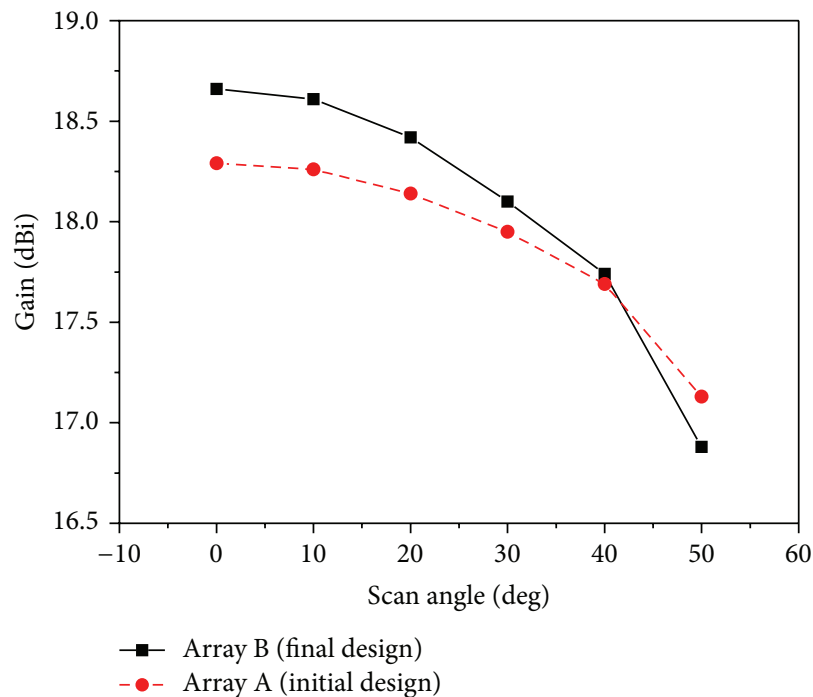

(a) Horizontal polarization

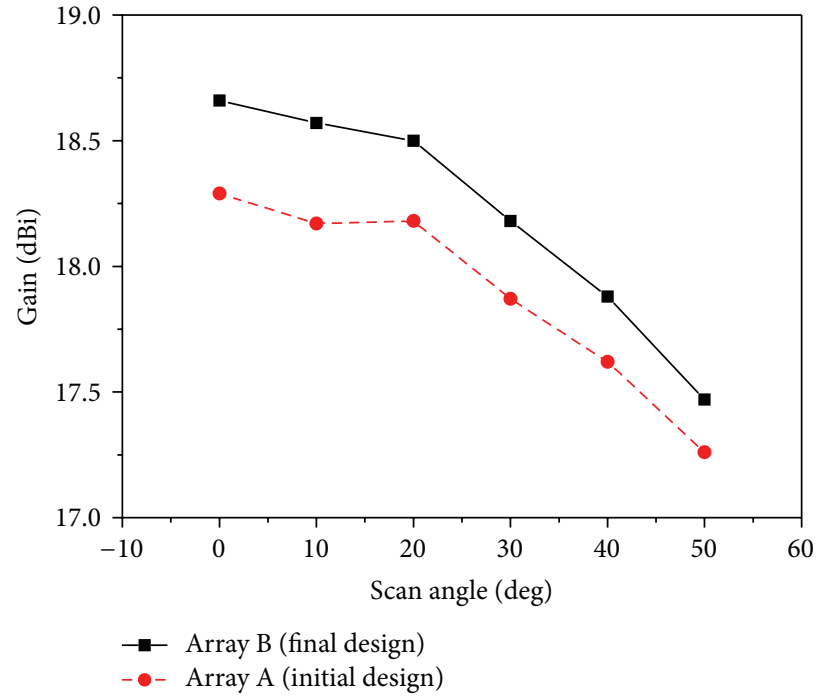

(b) Vertical polarization

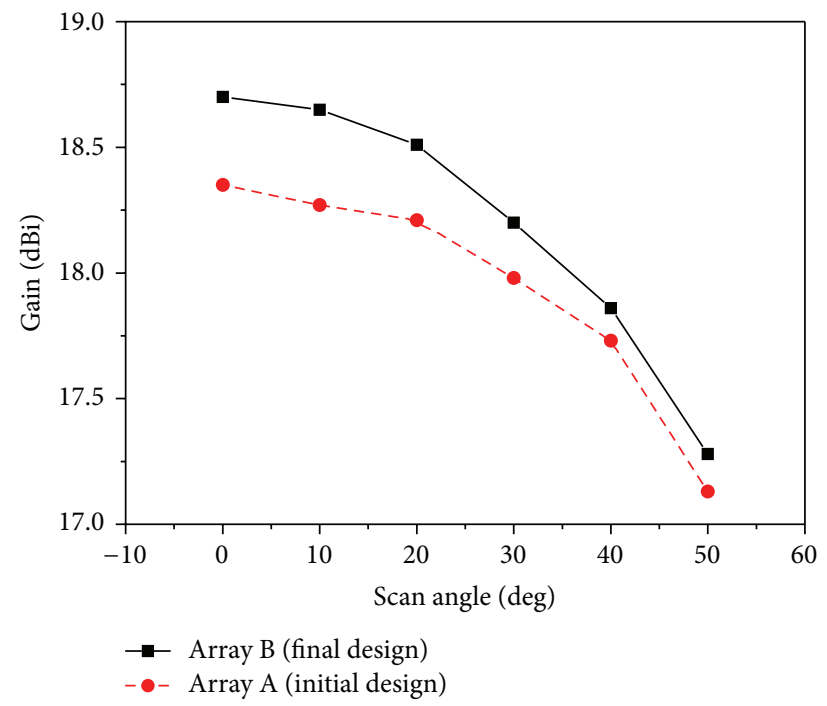

(c) Diagonal polarization

FIGURE 7: The comparison of gain during polarization tracking and beam scanning (azimuth angle $\varphi=0^{\circ}$ ).

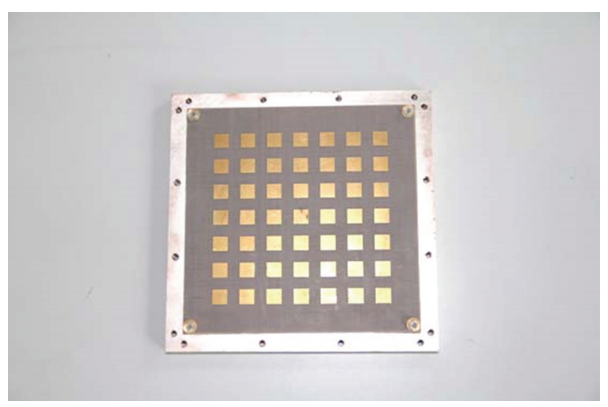

Figure 8: Front view of the fabricated array. 


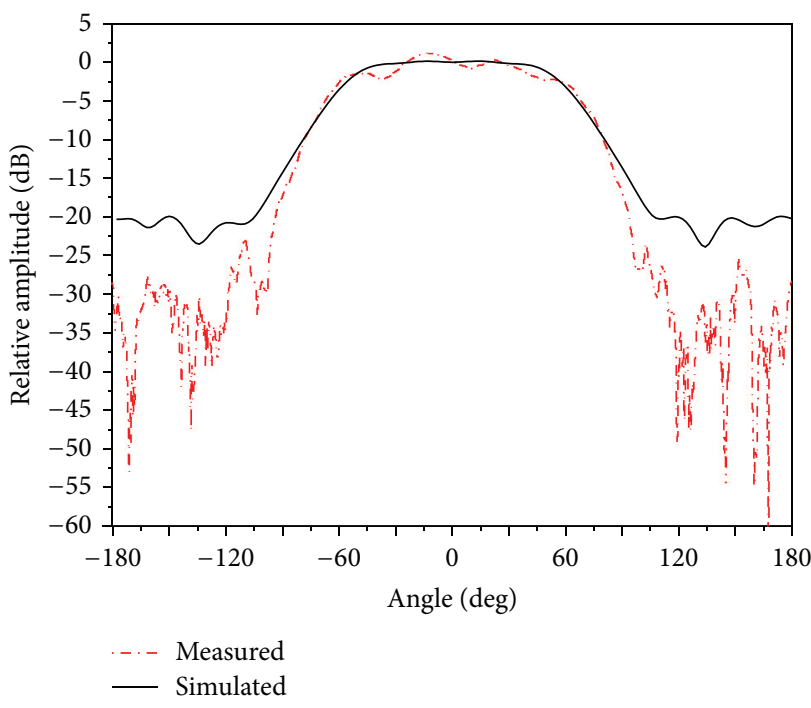

(a) $\varphi=0^{\circ}$ (H-plane)

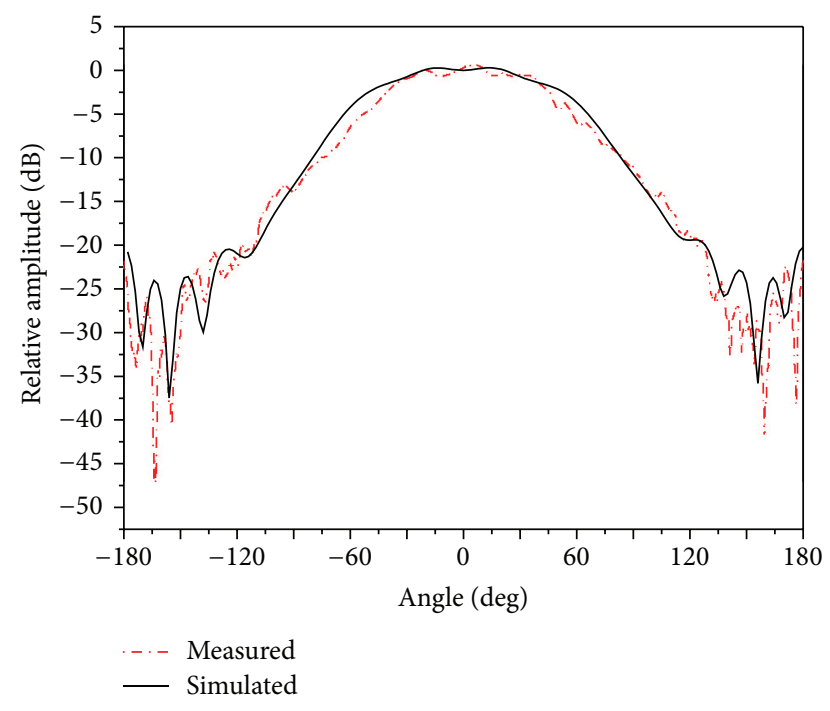

(b) $\varphi=90^{\circ}$ (E-plane)

Figure 9: The active element pattern of central element R5-3 fed from port V.

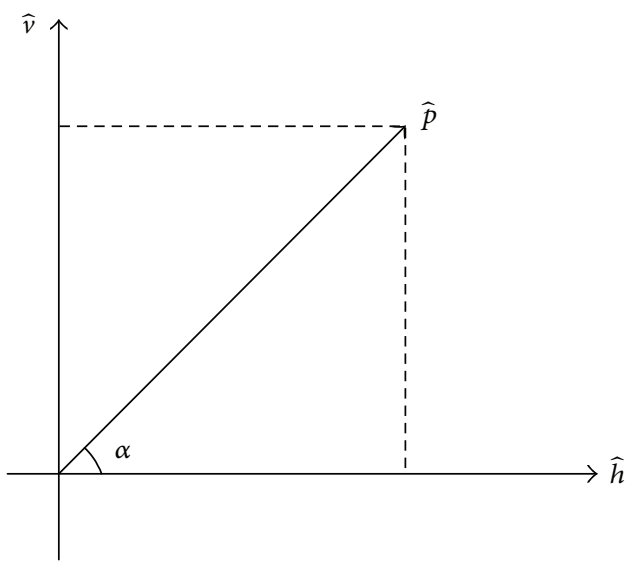

(a) Polarization decomposition

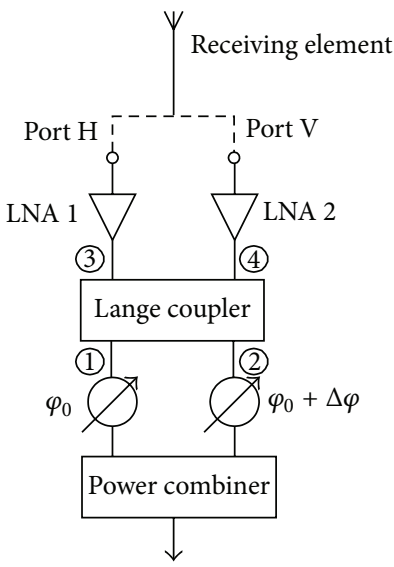

(b) Schematic block diagram

FIGURE 10: The polarization tracking module for receiving application.

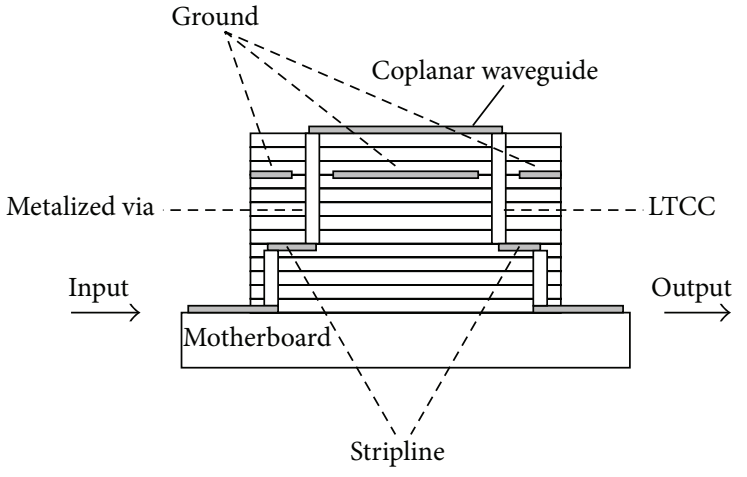

(a) Schematic diagram

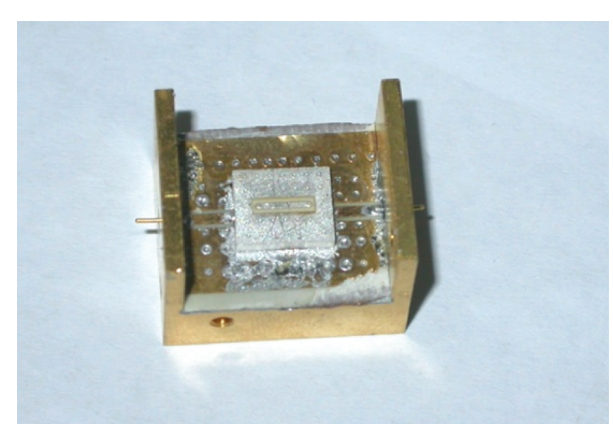

(b) Prototype

FIGURE 11: Transition from planar waveguide to stripline based on LTCC technology. 


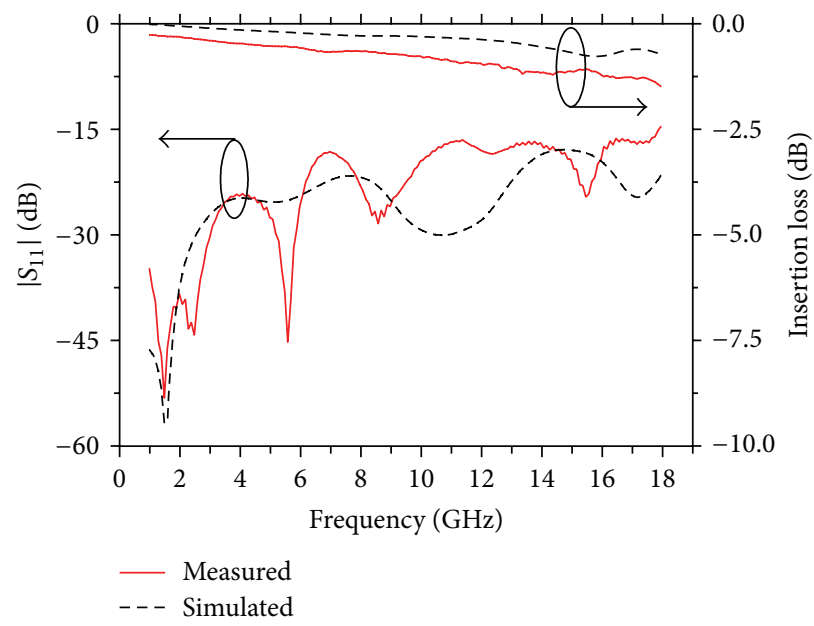

FIGURE 12: Reflection coefficient and insertion loss of the LTCC-based vertical transition.

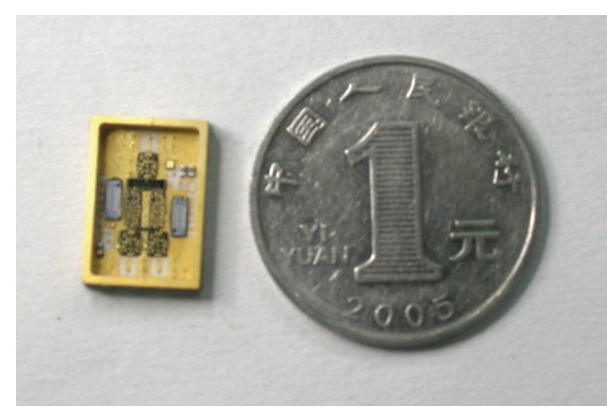

(a) Top view

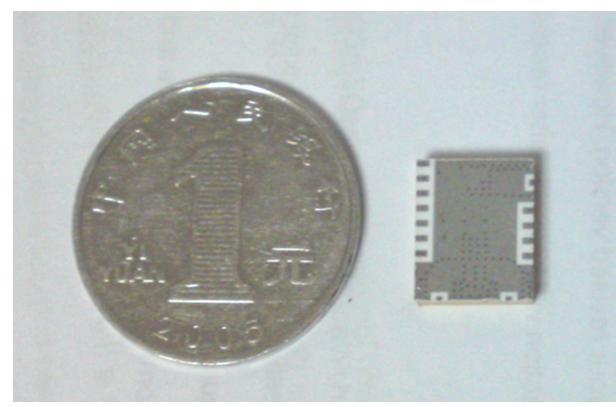

(b) Bottom view

FIGURE 13: The fabricated compact polarization tracking module.

measured reflection coefficient and insertion loss are plotted in Figure 12. It is observed that good agreement can be obtained between the simulated and measured results from $2 \mathrm{GHz}$ to $18 \mathrm{GHz}$. The measured reflection coefficient is below $-16 \mathrm{~dB}$, and the insertion loss is less than $1.5 \mathrm{~dB}$. We used the compact structure of the vertical transition to develop a polarization tracking module integrated with multiple MMICs, which is shown in Figure 13. With reference to Figure 10(b), the gain of two channels of the module was measured with $\varphi_{0}=0^{\circ}$ when $\Delta \varphi$ was increased from $0^{\circ}$ to $-360^{\circ}$ with a step of $-5.625^{\circ}$. With reference to Figure 14, the measured gain of both channels varies in the range from about $10 \mathrm{~dB}$ to $35 \mathrm{~dB}$ with different trend, which is determined by the differential phase $\Delta \varphi$ between two channels. Thus, the different amplitude weighted coefficients for both port $\mathrm{V}$ and port $\mathrm{H}$ can be achieved, which results in the arbitrary linear polarization of the antenna element. With reference to Figure 14, the diagonal polarization $\left(\alpha=45^{\circ}\right.$ and $\alpha=$ $-45^{\circ}$ ) can be obtained due to the approximately same gain of both channels with $\Delta \varphi=0^{\circ}$ and $-180^{\circ}$. The approximate vertical polarization can be obtained with $\Delta \varphi=-90^{\circ}$ when the measured gains of port $\mathrm{H}$ and port $\mathrm{V}$ are about $10.5 \mathrm{~dB}$ and $33.7 \mathrm{~dB}$. The approximate horizontal polarization can be obtained with $\Delta \varphi=-270^{\circ}$ when the measured gains of port $\mathrm{H}$ and port $\mathrm{V}$ are about $34.2 \mathrm{~dB}$ and $10.5 \mathrm{~dB}$.

\section{Experiment Result}

A small $7 \times 7$ polarization tracking active phased array was fabricated to cover the frequency range from $12.25 \mathrm{GHz}$ to $12.75 \mathrm{GHz}$. The inner $5 \times 5$ radiating elements are connected to the polarization tracking modules discussed above. The miniature $50 \Omega$ SMP female terminations are used as matched loads directly connecting to the outermost 24 passive radiating elements. The schematic block diagram of the fabricated array is shown in Figure 15. The compactness of the polarization tracking module based on LTCC$\mathrm{SiP}$ technology contributes to the low-profile phased array with the size of $120 \mathrm{~mm}$ (length) $\times 120 \mathrm{~mm}$ (width) $\times 55 \mathrm{~mm}$ (height). The polarization tracking patterns were measured in $x z$-plane. The antenna prototype to be measured was fixed on the rotary platform in the microwave chamber, and the polarization can be controlled electronically without rotating the array aperture. The patterns for horizontal, vertical, and diagonal polarization can be measured, respectively, with $\Delta \varphi=-270^{\circ},-90^{\circ}$, and $0^{\circ}$. The narrow bandwidth of $4 \%$ determines the stable radiation patterns over the entire operating frequency range from $12.25 \mathrm{GHz}$ to $12.75 \mathrm{GHz}$. Thus, the polarization tracking patterns measured at $12.5 \mathrm{GHz}$ with beam scanned to $0^{\circ}, 20^{\circ}, 40^{\circ}$, and $50^{\circ}$ are given in Figures 16, 17, 18, and 19. The reasonable agreement between 


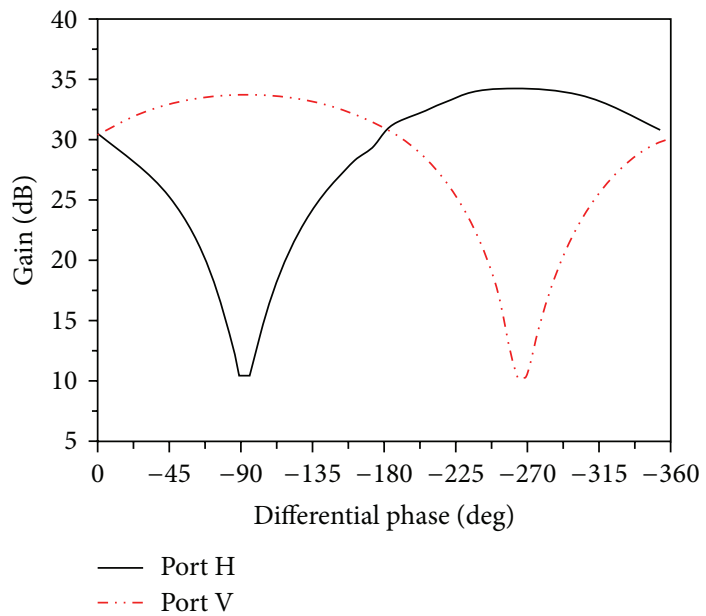

FIGURE 14: The measured transmission gain versus the differential phase $\Delta \varphi$ for port $\mathrm{H}$ and port V.

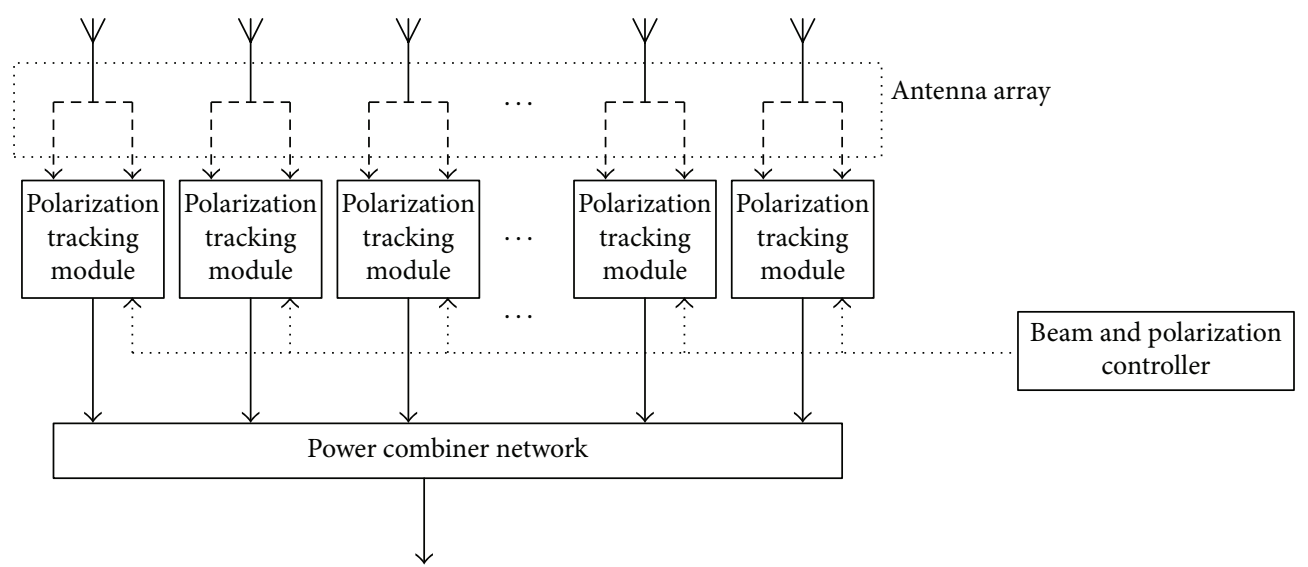

FIGURE 15: The schematic block diagram of the fabricated polarization tracking active phased array.

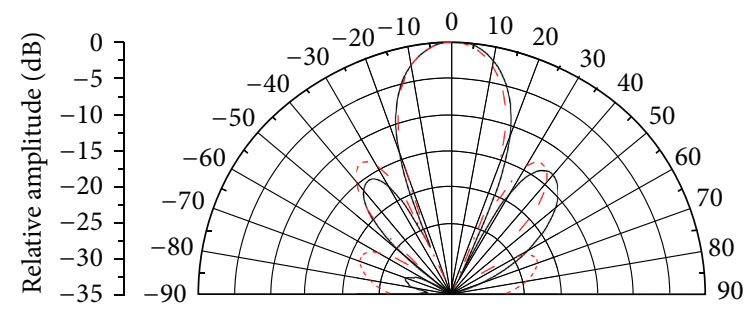

(a) Horizontal polarization
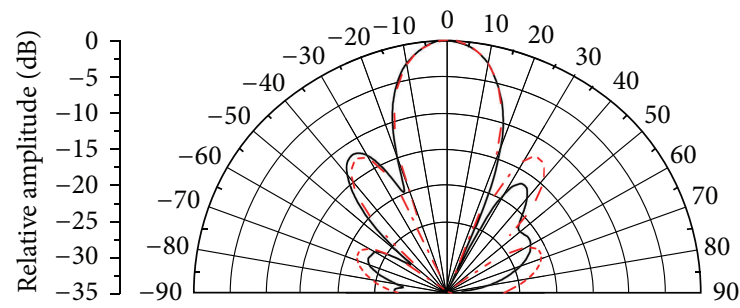

(b) Vertical polarization

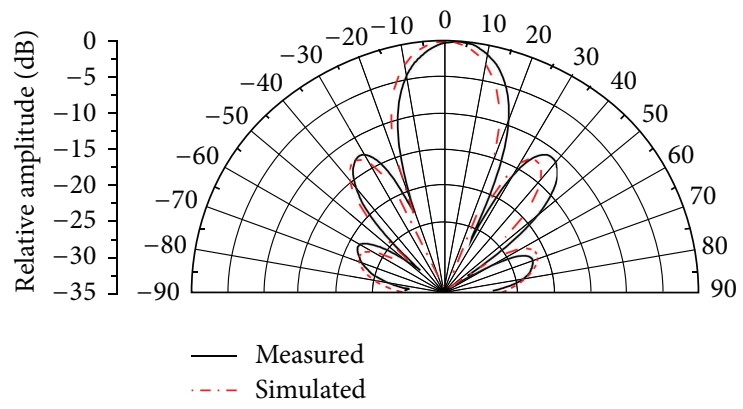

(c) Diagonal polarization

Figure 16: The polarization tracking patterns measured at $12.5 \mathrm{GHz}$ with beam scanned to $0^{\circ}$. 


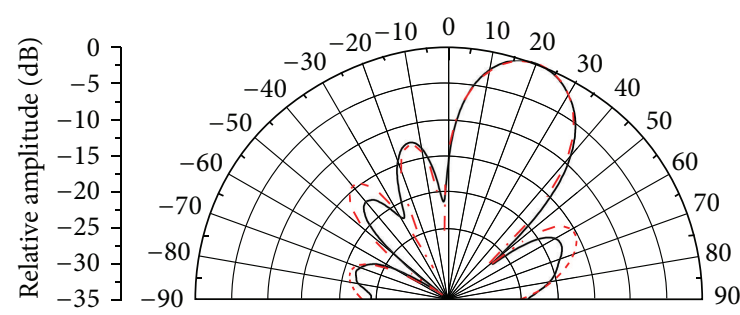

(a) Horizontal polarization

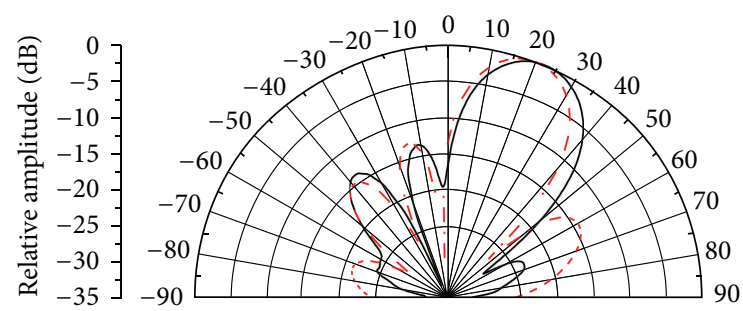

(b) Vertical polarization

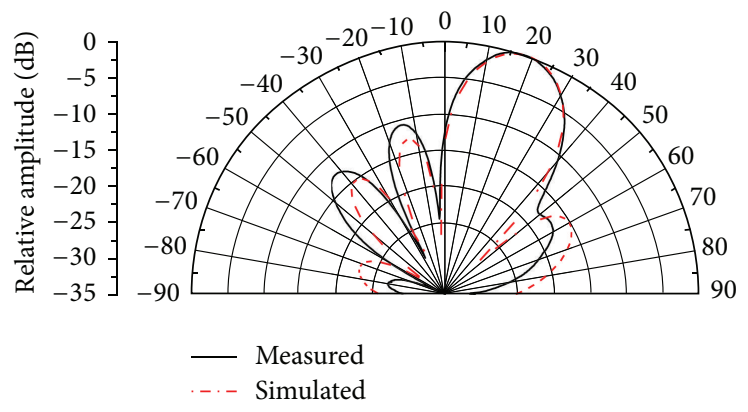

(c) Diagonal polarization

FIGURE 17: The polarization tracking patterns measured at $12.5 \mathrm{GHz}$ with beam scanned to $20^{\circ}$.

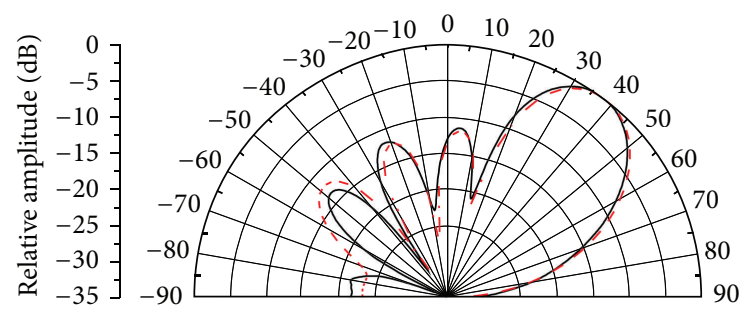

(a) Horizontal polarization

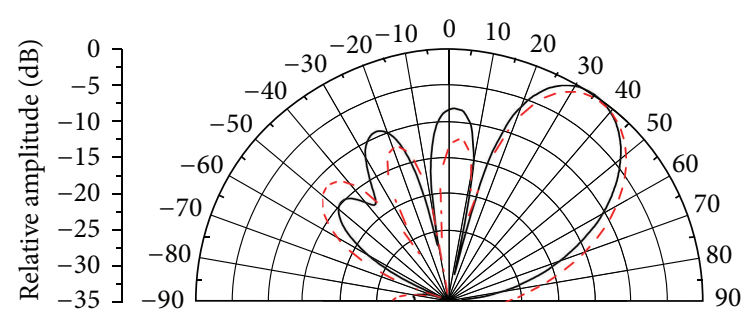

(b) Vertical polarization

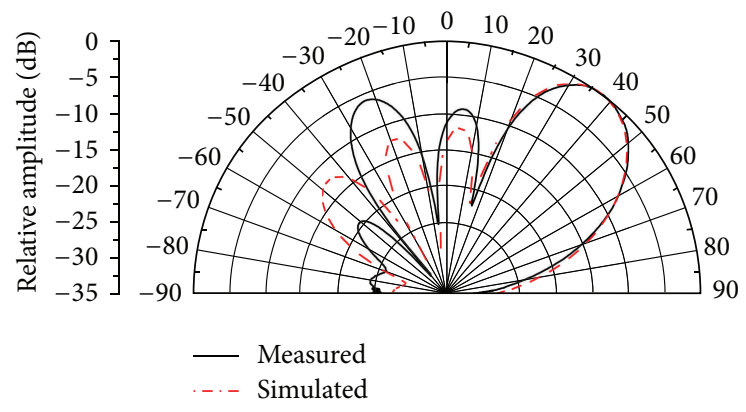

(c) Diagonal polarization

Figure 18: The polarization tracking patterns measured at $12.5 \mathrm{GHz}$ with beam scanned to $40^{\circ}$.

the simulated and measured results can be observed. The summary of the antenna prototype performance measured at $12.5 \mathrm{GHz}$ is provided in Table 2, wherein $\mathrm{H}, \mathrm{V}$, and $\mathrm{D}$ denote horizontal, vertical, and diagonal polarization. It is found that the mutual coupling results in the sidelobe level are greater than $-10 \mathrm{~dB}$ when the beam is steered to the angle greater than $40^{\circ}$. Furthermore, the beamwidth and beam pointing error increase. Table 2 gives the measured peak gain of the polarization tracking array prototype during beam scanning and polarization tracking. Compared with the simulated results, small errors exist. Since there are actually $5 \times 5$ active radiating elements in the small $7 \times 7$ array, the maximum directivity of the array is approximately $18.9 \mathrm{dBi}$. Thus, the aperture efficiency greater than $50 \%$ for the polarization tracking array prototype can be evaluated according to the measured peak gain. 


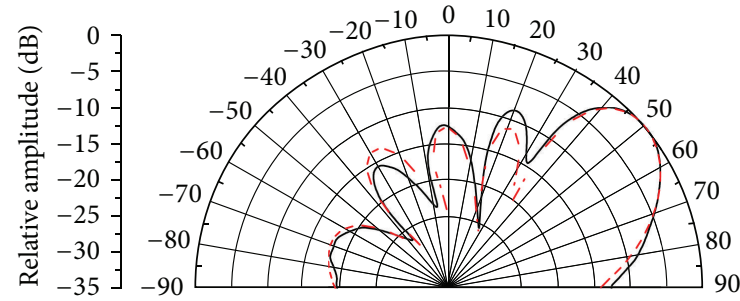

(a) Horizontal polarization

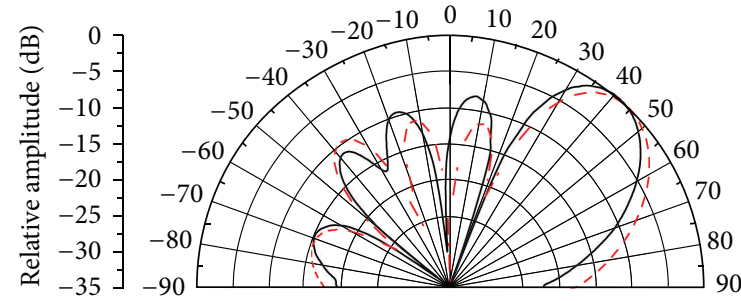

(b) Vertical polarization

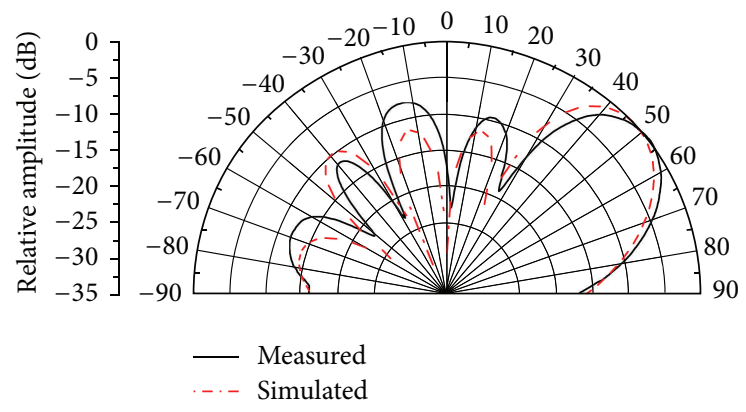

(c) Diagonal polarization

FIGURE 19: The polarization tracking patterns measured at $12.5 \mathrm{GHz}$ with beam scanned to $50^{\circ}$.

TABLE 2: The measured data at $12.5 \mathrm{GHz}$ for the developed small phased array prototype.

\begin{tabular}{lcccccccccccc}
\hline Desired scan angle (deg) & & $0^{\circ}$ & & \multicolumn{3}{c}{$20^{\circ}$} & \multicolumn{4}{c}{$40^{\circ}$} & \multicolumn{3}{c}{$50^{\circ}$} \\
& $\mathrm{H}$ & $\mathrm{V}$ & $\mathrm{D}$ & $\mathrm{H}$ & $\mathrm{V}$ & $\mathrm{D}$ & $\mathrm{H}$ & $\mathrm{V}$ & $\mathrm{D}$ & $\mathrm{H}$ & $\mathrm{V}$ & $\mathrm{D}$ \\
\hline Peak gain (dBi) & & & & & & & & & & & \\
$\quad$ Simulated & 18.7 & 18.7 & 18.7 & 18.4 & 18.5 & 18.5 & 17.7 & 17.9 & 17.9 & 16.9 & 17.5 & 17.3 \\
$\quad$ Measured & 18.3 & 18.5 & 18.2 & 17.9 & 17.7 & 18.0 & 17.2 & 17.4 & 17.5 & 16.1 & 16.3 & 16.2 \\
Aperture efficiency (\%) & 87 & 91 & 85 & 79 & 76 & 81 & 68 & 71 & 72 & 52 & 55 & 53 \\
SLL (dB) & -13.3 & -11.8 & -11.3 & -12.3 & -13.3 & -10.6 & -11.3 & -8.4 & -6.0 & -7.9 & -8.8 & -7.4 \\
Half-power beam width (deg) & $22.2^{\circ}$ & $21.3^{\circ}$ & $21.6^{\circ}$ & $21.9^{\circ}$ & 23.7 & $22.2^{\circ}$ & $24.3^{\circ}$ & $25.2^{\circ}$ & $24.3^{\circ}$ & $26.4^{\circ}$ & $24.9^{\circ}$ & $25.5^{\circ}$ \\
Beam pointing error (deg) & 0 & $-1.2^{\circ}$ & $3^{\circ}$ & $-1.4^{\circ}$ & $2.2^{\circ}$ & $-1.4^{\circ}$ & $-3.4^{\circ}$ & $-3.4^{\circ}$ & $-1.6^{\circ}$ & $-4.1^{\circ}$ & $-5.9^{\circ}$ & $1.3^{\circ}$ \\
\hline
\end{tabular}

\section{Conclusion}

We propose a compact polarization tracking active phased array for $\mathrm{Ku}$-band satellite communication. The phased array with the height of $55 \mathrm{~mm}$ is suited to be used in the application strictly limiting the profile of the antenna. Based on the simulated single radiating element, a small $7 \times 7$ array model was established with the simulation tool CST Microwave Studio. The outermost 24 elements are connected to $50 \Omega$ passive loads, which contributes to the $5 \times 5$ active array. The whole array was simulated, and the effects of mutual coupling on the impedance of the ports can be analyzed. Based on the dimensional parameters of the isolated antenna element, the parameters were adjusted via numerous simulations. The final design with mutual coupling considered had lower active reflection coefficient of $-15 \mathrm{~dB}$ with beam pointed to the boresight of the array. When the array operates in the states of horizontal, vertical, and diagonal polarization, the simulated active reflection coefficients versus the scan angle for central element are plotted. Furthermore, we propose a compact LTCC-based polarization tracking module based on a vertical transition from planar waveguide to stripline. In the frequency range from $12.25 \mathrm{GHz}$ to $12.75 \mathrm{GHz}$, a small polarization tracking active phased array prototype was fabricated, and its polarization can be configured electronically via the proposed modules. The measured polarization tracking patterns for horizontal, vertical, and diagonal polarization are given. It can be found from the measurement that the beam can be steered up to $50^{\circ}$ in the elevation, and the peak gain is more than $16.0 \mathrm{dBi}$. The aperture efficiency of more than $50 \%$ can be obtained. The experiment validates the availability of the array design with mutual coupling considered.

\section{References}

[1] S. Yamamoto, S. Nuimura, T. Mizuno, and Y. Inasawa, "A Ku band small reflector antenna using backfire primary radiator for satellite communication system on board vessel," in Proceedings of the International Symposium on Antennas and Propagation (ISAP '12), pp. 1273-1276, Nagoya, Japan, October 2012. 
[2] J. Thornton, A. White, and G. Long, "Multi-beam scanning lens antenna for satellite communications to trains," Microwave Journal, vol. 52, no. 8, pp. 56-70, 2009.

[3] A. R. Weily and N. Nikolic, "Dual-polarized planar feed for lowprofile hemispherical Luneburg lens antennas," IEEE Transactions on Antennas and Propagation, vol. 60, no. 1, pp. 402-407, 2012.

[4] S. H. Son and U. H. Park, "Sidelobe reduction of low-profile array antenna using a genetic algorithm," ETRI Journal, vol. 29, no. 1, pp. 95-98, 2007.

[5] P. Mousavi, M. Fakharzadeh, S. H. Jamali et al., "A low-cost ultra low profile phased array system for mobile satellite reception using zero-knowledge beamforming algorithm," IEEE Transactions on Antennas and Propagation, vol. 56, no. 12, pp. 36673679, 2008.

[6] S. Vaccaro, F. Tiezzi, M. F. Rúa, and C. D. G. De Oro, "Ku-Band Low-Profile Rx-only and Tx-Rx antennas for mobile satellite communications," in Proceedings of the 4th IEEE International Symposium on Phased Array Systems and Technology (Array '10), pp. 536-542, Waltham, Mass, USA, October 2010.

[7] R. V. Gatti, L. Marcaccioli, E. Sbarra, and R. Sorrentino, "Flat array antennas for Ku-band mobile satellite terminals," International Journal of Antennas and Propagation, vol. 2009, Article ID 836074, 5 pages, 2009.

[8] C. O. Adler, A. D. Monk, D. N. Rasmussen, and M. J. Taylor, "Two-way airborne broadband communications using phased array antennas," in Proceedings of the IEEE Aerospace Conference, vol. 2, pp. 925-922, March 2003.

[9] S. Hasegawa, T. Yasuzumi, O. Hashimoto, and Y. Kazama, "Polarization tracking phased array antenna with cross dipole antenna-measured results," in Proceedings of the IEEE International Symposium on Antennas and Propagation, Toronto, Canada, July 2010.

[10] J. Zhou, W. Shi, W. B. Dou, and Y. Shen, "High integrated microwave architecture using LTCC-SIP technology in active phased array antenna applications," Frequenz, vol. 66, pp. 177182, 2012. 

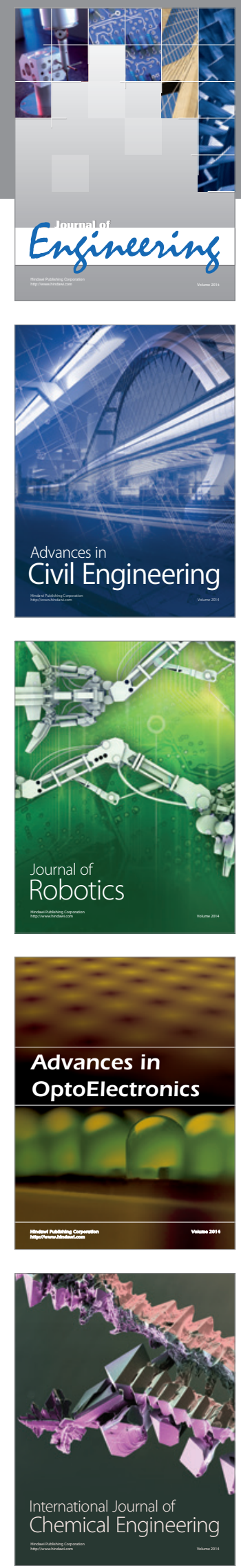

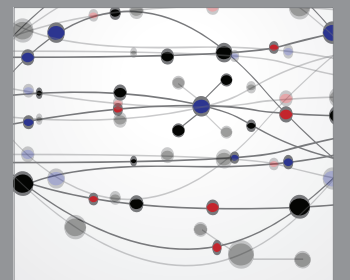

The Scientific World Journal
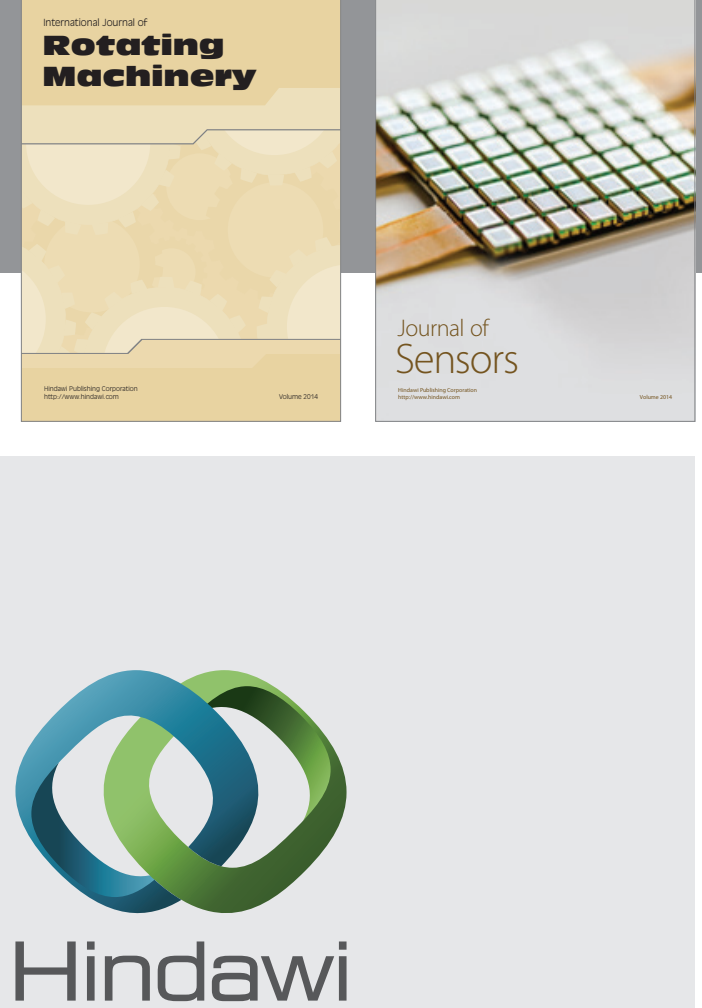

Submit your manuscripts at http://www.hindawi.com
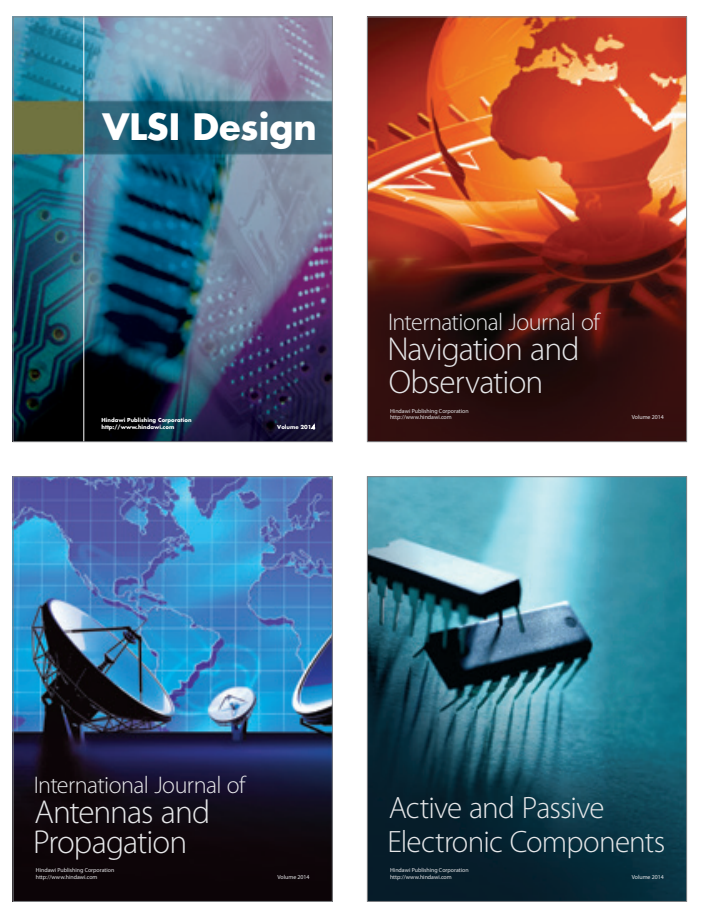
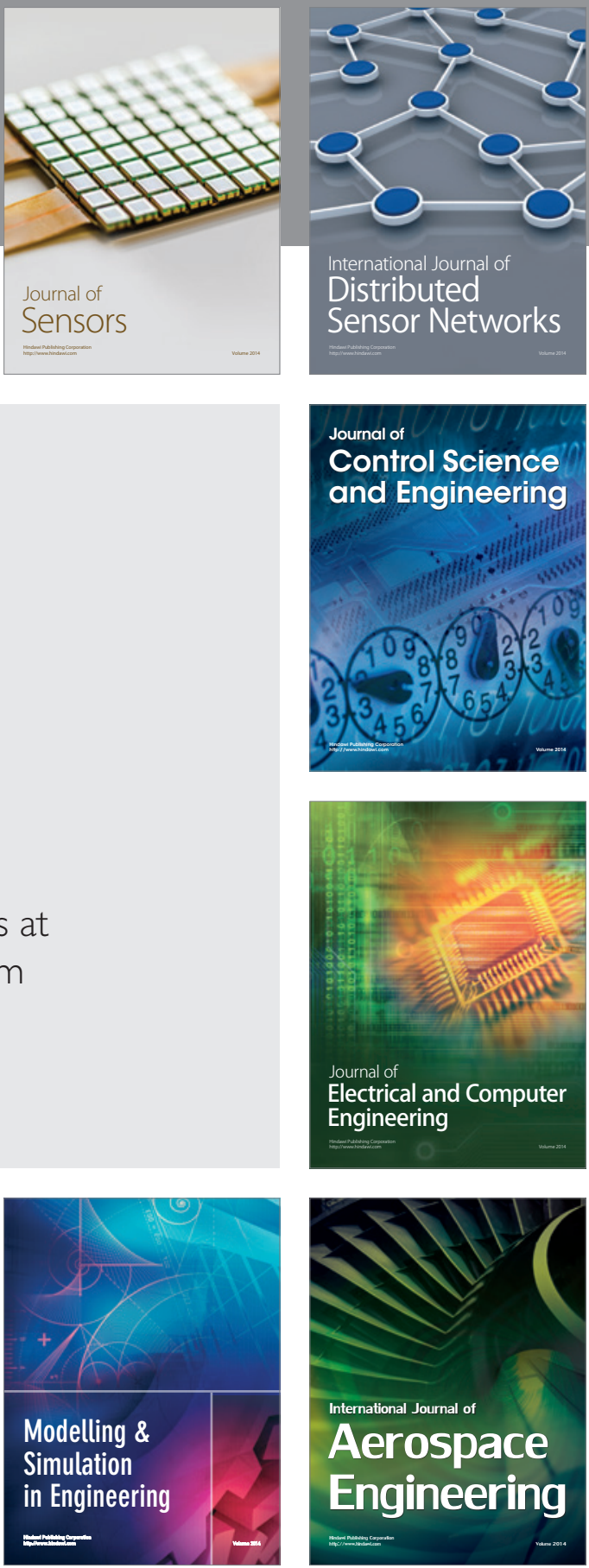

Journal of

Control Science

and Engineering
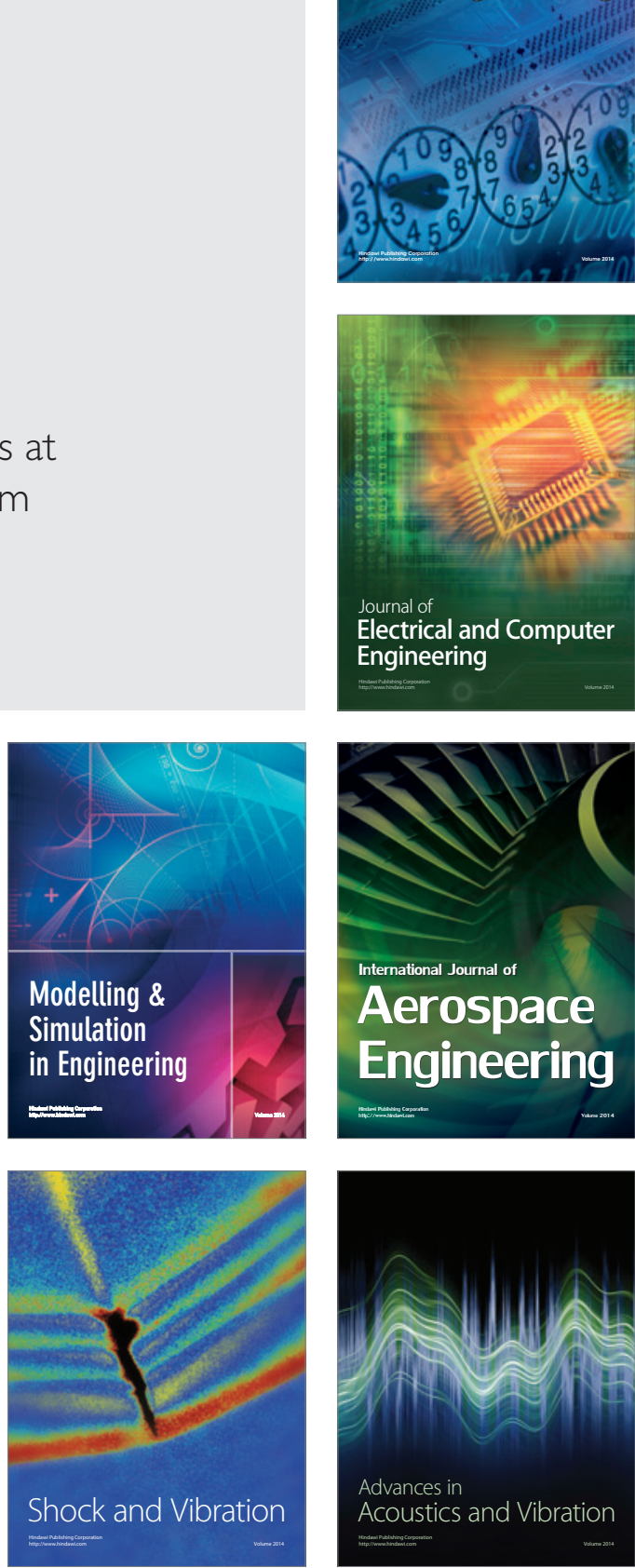